\title{
EFECTO DE LA ALTURA DE CORTE EN EL REBROTE DE Acacia saligna (Labill.) Wendl.
}

\author{
MAURICIO BRATTI R.(*), ANTONIO VITA A.(**) y JOHANNES WRANN H.(**) \\ (*) Ingeniero Forestal, INFOR. \\ (**) Ingeniero Forestal, Universidad de Chile.
}

\section{RESUMEN}

El estudio se realizó en una plantación de Acacia saligna ubicada en la localidad de llia, Illapel, IV Región, con el propósito de determinar el efecto de cortes horizontales efectuados a tres alturas del árbol (corte a menos de $5 \mathrm{~cm}$, a $50 \mathrm{~cm}$ y a $100 \mathrm{~cm}$ ), sobre la biomasa de rebrote palatable al ganado. El período de esta evaluación fue de una temporada. En el análisis efectuado se consideró la altura original del árbol para determinar si ésta tiene alguna influencia en el desarrollo posterior del rebrote. Además, se analizó la productividad obtenida en el rebrote para los tratamientos propuestos.

Los principales resultados indicaron que los ejemplares cortados a $50 \mathrm{~cm}$ de altura se diferenciaron significativamente, en cuanto a vigor y crecimiento, de las otras alturas de corte realizadas. Sobre la base de las conclusiones obtenidas, se recomienda intervenir la plantación tempranamente y de esta manera lograr una mayor productividad en comparación a la proveniente de ejemplares sin ninguna intervención.

Palabras Clave: Acacia, corte, rebrote.

\section{ABSTRACT}

The study carried out in a plantation of Acacia saligna located in the area of Ilta, Illapel, IV Region.The purpose of the study was to determine the effect of horizontal prunning at three different heights of the tree (less than $5 \mathrm{~cm}, 50 \mathrm{~cm}$ and $100 \mathrm{~cm}$ ) over the fodder biomass. The period for the assesement was a season. It was also considered the original tree height to determine its influence over the fodder biomass production. Besides to that, productivity versus proposed treatments was analysed.

The most revelant results show that trees cut at $50 \mathrm{~cm}$ height were more vigorous and with better growth than those cut al less than $5 \mathrm{~cm}$ or at $100 \mathrm{~cm}$ height. Considering the conclusions of the study, early interventions are recomended thus obtaining greater productivity compared to trees without intervention.

Keywords: Acacia, prunning, regrowth 


\section{INTRODUCCIÓN}

La deforestación causada por el uso indiscriminado de la leña y el carbón para satisfacer las necesidades básicas de los hogares, principalmente para la cocción de alimentos, constituye en la actualidad motivo de gran preocupación. Los perjuicios que se derivan de estas prácticas son enormes, ya sea desde un punto de vista ambiental como en términos socio-económicos: pérdida de suelos, biodiversidad y elevados costos de tiempo y dinero en el abastecimiento de energía (FAO, 1992).

El desbalance entre oferta y demanda de leña puede causar un efecto irreparable a la vegetación y al medio ambiente, especialmente en áreas ecológicamente frágiles, como son las zonas áridas.

La rehabilitación de terrenos degradados a través de la forestación, constituye una herramienta eficaz para poner a disposición de la población rural biomasa para la obtención de energía, forraje y otros productos de primera necesidad. De esta manera, se puede contribuir en forma significativa al desarrollo de este sector de la población, el cual presenta serios problemas, incluso para satisfacer sus necesidades básicas.

Una vez instaladas las plantaciones, es necesario aplicar tratamientos silviculturales con el propósito de obtener arquitecturas de los ejemplares que permitan cumplir en mejor forma con los objetivos de la forestación.

En el caso de las especies arbóreas destinadas principalmente a forraje, dado el tamaño excesivo que los ejemplares adultos pueden alcanzar, la mayor proporción de su follaje queda fuera del alcance del ganado. Esto ocasiona una pérdida evidente de alimento y, por otra parte, un envejecimiento del arbusto. Además de su tamaño excesivo, las ramas ya lignificadas son poco flexibles, lo cual hace que éstas sean frecuentemente desganchadas por animales que tratan de alcanzar el forraje, provocando un deterioro del recurso (LAILHACAR, 1986). El corte del árbol a temprana edad permite al animal hacer más accesible la vegetación proveniente del rebrote, estimulando además la producción de tejidos tiernos más nutritivos para el ganado.

La especie Acacia saligna se ha incluido en una serie de ensayos de introducción de especies en la zona árida de Chile, en los cuales se ha alcanzado excelentes resultados de sobrevivencia y desarrollo (VITA y GREZ, 1992). Por tal motivo, a través del presente estudio se pretendió obtener pautas de manejo silvicultural para la especie, de acuerdo a los requerimientos de la población del sector y de este modo, contribuir al combate del proceso de desertificación que afecta a dicha zona.

Por consiguiente, el objetivo general de este estudio fue determinar la respuesta de Acacia saligna frente a un corte temprano a diferentes alturas en ejemplares de distintos tamaños originales. Asimismo, cuantificar la biomasa potencialmente forrajera obtenida como respuesta a los tratamientos. 


\section{MATERIAL Y MÉTODO}

\section{Descripción del Área Experimental}

El estudio se desarrolló en la Estación Experimental Ilta perteneciente al Instituto Forestal, utilizando una plantación de dos años de Acacia saligna. El área se encuentra en la región de clima mediterráneo árido. Está ubicada a $33 \mathrm{~km}$ al norte de la ciudad de Illapel, en la Comuna de Los Vilos, Provincia de Choapa, IV Región, a $31^{\circ} 37$ 'S y $71^{\circ}$ 16'O. La plantación se encuentra a una altitud de $450 \mathrm{~m}$ y tiene una superficie aproximada de 0,5 ha. Se ubica sobre la vertiente oriental de la Cordillera de la Costa, formando parte del "secano interior" (WRANN, 1985).

El clima se caracteriza por una temperatura media anual de alrededor de $15^{\circ} \mathrm{C}$, con una gran amplitud diaria $\left(18\right.$ a $\left.20^{\circ} \mathrm{C}\right)$, y por una humedad relativa más bien baja $(50 \%$ promedio anual). Las precipitaciones son variables, pero en general, inferiores a $250 \mathrm{~mm}$ anuales, concentradas en un $88 \%$ en otoño e invierno (WRANN, 1985). El período libre de heladas es de 10 a 11 meses, con dos meses de receso vegetativo.

El suelo del área experimental corresponde a aridisoles de origen granítico de transición entre pardo cálcicos a pardo no cálcicos, de profundidad media y textura franco arcillosa.

La vegetación natural existente en el área experimental corresponde a una formación arbustiva baja dominada por Gutierrezia paniculata, con algunos ejemplares de Flourensia thurifera, Colletia spinosa, Puya spp. y Cereaus spp.

\section{Instalación de la Plantación}

La plantación se realizó a un distanciamiento de 4 × $4 \mathrm{~m}$ en un suelo previamente subsolado. Tres semanas después de la plantación se aplicó a cada ejemplar un kilogramo de guano de cabra. Simultáneamente, se limpió la taza de plantación en forma manual, abarcando un metro de diámetro. Por otra parte, se aplicaron riegos mensuales durante el período seco del primer año y riegos de emergencia en años posteriores.

\section{Evaluación de la Respuesta de la Especie a Tres Alturas de Corte según Dos Clases de Altura Original de los Ejemplares Intervenidos}

En septiembre de 1993 se midió la altura total de los 160 árboles seleccionados al azar para el estudio (40 por tratamiento). Al mismo tiempo, se aplicaron los tratamientos mediante un corte horizontal con sierra de arco a 5,50 y $100 \mathrm{~cm}$ a partir del suelo. Posteriormente, en mayo de 1994, se midió, entre otras variables, el largo de cada rebrote, para luego obtener un largo promedio del rebrote por cada árbol. Además, se obtuvo el peso verde del rebrote en cada tratamiento para cuantificar la biomasa obtenida por cada uno de éstos. 
El diseño experimental para determinar la mejor respuesta de la especie frente a los tratamientos aplicados utilizó un modelo completamente aleatorizado de la forma:

$$
Y_{i j}=u+T_{i}+b * X_{i}+E_{i j}
$$

donde:

$T=$ tratamiento (altura de corte).

$\mathrm{X}=$ altura original de la planta.

$\mathrm{Y}=$ longitud media del rebrote.

$\mathrm{u}=$ promedio de la altura de corte.

$\mathrm{b}=$ constante de la ecuación.

Los datos se estudiaron a través de un análisis de covarianza, en que el modelo matemático utilizado fue el siguiente:

$$
\begin{aligned}
& Y_{i j}=u+T_{i}+b X_{i}+E_{i j} \quad \mathrm{i}=1, \ldots, 4 \text { (tratamientos) } \\
& \mathrm{j}=1, \ldots, 40 \text { (repeticiones) }
\end{aligned}
$$

$\mathrm{T}=$ tratamiento (altura de corte)

$\mathrm{X}=$ altura original de la planta.

$\mathrm{Y}=$ longitud media del rebrote.

b $=$ constante del modelo.

$\mathrm{e}=$ error experimental.

$\mathrm{u}=$ promedio del modelo.

Posteriormente se aplicó la prueba $\mathrm{F}$ de la varianza y la prueba de rango múltiple de Duncan.

\section{Análisis de la Biomasa Obtenida en el Rebrote para cada Altura de Corte según Clase de Altura}

Para analizar la respuesta de los tratamientos en términos de biomasa, se seleccionaron 30 árboles, 15 de cada clase de altura. Dentro de cada clase de altura se extrajo la biomasa total de 5 individuos por tratamiento, seleccionando árboles promedio con el fin de obtener una muestra representativa. De esta manera, se analizó la producción de biomasa por tratamiento y clase de altura. Es necesario aclarar que se consideró la biomasa obtenida como respuesta de las tres intervenciones realizadas anteriormente (alturas de corte), por lo que el tratamiento testigo no aparece en el análisis de este punto.

\section{Recuperación al Corte por Tratamiento}

La recuperación al corte que presentaron los árboles intervenidos, se midió como un porcentaje de la altura inicial que tenía el árbol con respecto a la altura que éste alcanzó en la temporada siguiente a la intervención. 


\section{RESULTADOS Y DISCUSIÓN}

A continuación se presentan los principales resultados de este estudio.

\section{Biomasa Obtenida en el Rebrote según Altura de Corte y Clase de Altura}

Biomasa del Rebrote por Tratamiento

En el Cuadro 1 se muestra la biomasa obtenida en el rebrote para cada altura de corte. Según se aprecia en el cuadro, de las tres alturas de corte probadas en este estudio, el corte a $50 \mathrm{~cm}$ resultó ser la intervención en que se obtuvo la mayor productividad. Es preciso señalar que la mayor diferencia se presentó en la clase de altura más alta. Este resultado fue esperado si se considera que árboles con mayor vigorosidad, dominantes dentro de la plantación, son capaces de responder mejor a la intervención practicada.

Considerando las tres alturas de corte practicadas en la plantación, el corte a $50 \mathrm{~cm}$ presentó diferencias significativas al $95 \%$ de confianza con respecto a las otras alturas de corte, por lo que puede inferirse que este tratamiento es el de mejor resultado.

\section{Cuadro 1}

\section{PRODUCTIVIDAD DE LA PLANTA EN REBROTE POR TRATAMIENTO}

\begin{tabular}{|c|c|c|}
\hline $\begin{array}{l}\text { Altura de Corte } \\
\text { (cm) }\end{array}$ & \multicolumn{2}{|c|}{$\begin{array}{c}\text { Promedio } \\
\text { (kg/planta) }\end{array}$} \\
\hline 5 & 0,621 & $\mathrm{~b}$ \\
\hline 50 & 1,768 & $\mathrm{a}$ \\
\hline 100 & 0,988 & b \\
\hline
\end{tabular}

- Letras minúsculas distintas en una misma columna indican diferencias significativas entre las alturas de corte $(\mathrm{P}<=0,05)$.

GARCIA (1992), en su estudio con Atriplex nummularia obtuvo la mejor respuesta con la altura de corte a $50 \mathrm{~cm}$, alcanzando un promedio de $816 \mathrm{~g} /$ planta. En el caso de Acacia saligna la mejor respuesta de los tres tratamientos utilizados también se logró con el corte a $50 \mathrm{~cm}$, y el promedio fue más del doble que en el caso anterior.

Biomasa del rebrote por clase de altura

Al analizar la biomasa proveniente del rebrote de cada clase de altura, independientemente de las alturas de corte probadas, se obtienen los resultados que se indican en el Cuadro 2. 


\section{Cuadro 2}

\section{PRODUCTIVIDAD DE LA PLANTA EN REBROTE POR CLASE DE ALTURA}

\begin{tabular}{|c|cc|}
\hline $\begin{array}{c}\text { Clase de Altura } \\
\text { (m) }\end{array}$ & $\begin{array}{c}\text { Promedio } \\
\text { (kg/planta) }\end{array}$ \\
\hline $1,25-1,8$ & 0,813 & $\mathrm{a}$ \\
\hline $1,81-3,5$ & 1,439 & $\mathrm{a}$ \\
\hline
\end{tabular}

- Letras minúsculas distintas en una misma columna indican diferencias significativas entre las alturas de corte $(\mathrm{P}<=0,05)$.

Según el análisis estadístico, la biomasa del rebrote no presentó diferencias significativas $(\mathrm{P}<=0,05)$ entre las dos clases de alturas definidas. Esto se puede explicar por la excelente respuesta que se obtuvo con el corte a $50 \mathrm{~cm}$ en la clase de altura menor, lo que provocó un aumento en el promedio del peso seco, disminuyendo la diferencia con la otra clase de altura.

Para este análisis, GARCIA (1992), en las tres clases de altura que definió obtuvo como promedio en el rebrote de Atriplex nummularia $307,1 \mathrm{~g} /$ planta. Con Acacia saligna el promedio obtenido considerando las dos clases de altura fue de $1.126 \mathrm{~g} / \mathrm{planta}$. Cabe señalar que el período considerado en este estudio para realizar la evaluación de la intervención fue de sólo ocho meses (septiembre-mayo).

\section{Porcentaje de Recuperación al Corte por Tratamiento}

En el Cuadro 3 se indican las diferencias obtenidas en la respuesta al corte para las tres alturas de corte utilizadas.

Cuadro 3.

RECUPERACIÓN AL CORTE POR TRATAMIENTO

\begin{tabular}{|c|c|}
\hline $\begin{array}{c}\text { Altura de Corte } \\
(\mathrm{cm})\end{array}$ & $\begin{array}{c}\text { Recuperación } \\
(\%)\end{array}$ \\
\hline 5 & 57,2 \\
\hline 50 & 83,7 \\
\hline 100 & 94,5 \\
\hline
\end{tabular}

Considerando sólo la altura del árbol antes y después de la intervención, el porcentaje de recuperación al corte aumenta en conjunto con la altura de corte. Esto se podría explicar por la significación de las reservas almacenadas en tallos gruesos y finos, y por la inducción de yemas que se produce por la intervención (GARCIA, 1992). De esta manera, el corte a $100 \mathrm{~cm}$ presenta el mayor porcentaje de recuperación, debido a la mayor cantidad de brotes que se producen a lo largo de las ramas gruesas y finas que 
quedan al descubierto después de la intervención. Esto no ocurre con el corte a menos de $5 \mathrm{~cm}$, el que presenta el más bajo porcentaje de recuperación.

Sin embargo, el mayor porcentaje de recuperación al corte que presentó el corte alto con respecto a los otros tratamientos, no implica que la cantidad de biomasa obtenida sea también mayor. El porcentaje de recuperación al corte se midió considerando sólo la altura inicial y la obtenida en la segunda medición después de la intervención. De esta manera, en el corte a $100 \mathrm{~cm}$ de altura existe una menor diferencia con respecto a la altura original del árbol en comparación con el corte a 50 y $5 \mathrm{~cm}$; es decir, el tratamiento intermedio está más distante de la altura original del árbol que el tratamiento de corte alto y, a su vez, el tratamiento de corte bajo también lo está de los otros dos.

En el corte a $50 \mathrm{~cm}$ se observó un mayor rebrote y crecimiento lateral de los individuos, adoptando un hábito más arbustivo que el que tenían originalmente. De esta forma la recuperación en biomasa que se produjo no se vió reflejada en su totalidad en el porcentaje de recuperación al corte.

Cabe señalar que la mortalidad de individuos producto de los cortes fue mínima, alcanzando el $7,5 \%$ en el corte hecho a ras de suelo. En los restantes tratamientos no se observó mortalidad, comprobándose la excelente reacción que presenta Acacia saligna frente a este tipo de intervención.

Por otro lado, según Vita y Grez (1992), en ensayos realizados en condiciones de secano en la IV Región, Acacia saligna resultó ser la especie de más rápido crecimiento, con un incremento medio anual en altura que varía entre 30 y $71 \mathrm{~cm}$, sin practicar ningún tipo de intervención silvicultural. En el presente estudio se obtuvieron crecimientos superiores en los tratamientos uno y dos, tal como se indica en el Cuadro 4.

Cuadro 4

INCREMENTO EN ALTURA PROMEDIO POR TRATAMIENTO PARA UNA TEMPORADA DE OCHO MESES

\begin{tabular}{|c|c|}
\hline Altura de corte & $\begin{array}{c}\text { Incremento en altura } \\
\text { (cm) }\end{array}$ \\
\hline $5 \mathrm{~cm}$ & 75,3 \\
\hline $50 \mathrm{~cm}$ & 101,3 \\
\hline $100 \mathrm{~cm}$ & 68,3 \\
\hline testigo & 38,4 \\
\hline
\end{tabular}

\section{Efecto de la Altura de Corte en el Largo Medio del Rebrote de la Planta}

Tal como se menciona en la metodología, en este punto se analizó la información mediante dos formas:

a. Análisis de Covarianza: Se utilizó como variable dependiente al largo medio del rebrote (LMR) y como variable independiente a la altura total del árbol (ATOT). Se consideraron 160 pares de datos, 40 por tratamiento. 
Los resultados se presentan a continuación:

Cuadro 5.

ANÁLISIS TOTAL DEL REBROTE

\begin{tabular}{|c|cc|}
\hline Altura de corte & $\begin{array}{c}\text { Largo medio del } \\
\text { rebrote } \\
\text { (LMR) }\end{array}$ \\
\hline $50 \mathrm{~cm}$ & 1,0130 & $\mathrm{~A}$ \\
\hline $5 \mathrm{~cm}$ & 0,7526 & $\mathrm{~B}$ \\
\hline $100 \mathrm{~cm}$ & 0,6827 & $\mathrm{~B}$ \\
\hline testigo & 0,3840 & $\mathrm{C}$ \\
\hline
\end{tabular}

Con el corte a $50 \mathrm{~cm}$ se obtuvo una longitud del rebrote significativamente mayor que en las otras alturas de corte probadas. Las alturas de corte a menos de $5 \mathrm{~cm}$ y $100 \mathrm{~cm}$ no mostraron diferencias significativas entre sí, pero éstas se diferenciaron del tratamiento testigo, con el cual se obtuvieron los valores más bajos de crecimiento de la planta en el período estudiado (una temporada).

De acuerdo a observaciones de terreno, podría decirse que individuos que en la condición original presentaban una mayor cantidad de brotes desde la base, o en sus ramas laterales, tuvieron un rebrote más abundante.

b. Análisis de Varianza: El análisis de los datos se hizo en forma separada, por clase de altura, de manera de evitar el error producido por la heterogeneidad de los valores de la variable independiente (altura total del árbol).

\section{Cuadro 6}

ANÁLISIS DEL REBROTE EN LA CLASE DE ALTURA 1 (1,25 - 1,80 m).

\begin{tabular}{|c|cc|}
\hline Altura de corte & $\begin{array}{c}\text { Largo medio del rebrote } \\
(\text { LMR })(\mathbf{m})\end{array}$ \\
\hline $50 \mathrm{~cm}$ & 0,7430 & $\mathrm{~A}$ \\
\hline $5 \mathrm{~cm}$ & 0,6846 & $\mathrm{~A}$ \\
\hline $100 \mathrm{~cm}$ & 0,5850 & $\mathrm{~A}$ \\
\hline testigo & 0,2690 & $\mathrm{~b}$ \\
\hline
\end{tabular}

Las alturas de corte probadas no mostraron diferencias significativas entre sí, pero el testigo presentó un crecimiento significativamente menor en relación a las intervenciones practicadas. 
Cuadro 7

\section{ANÁLISIS DEL REBROTE EN LA CLASE DE ALTURA 2 (1,81 - 3,5 m)}

\begin{tabular}{|c|cc|}
\hline Altura de corte & \multicolumn{2}{|c|}{$\begin{array}{c}\text { Largo medio del rebrote } \\
(\text { LMR })(\mathrm{m})\end{array}$} \\
\hline $50 \mathrm{~cm}$ & 1,3463 & A \\
\hline $100 \mathrm{~cm}$ & 0,7834 & B \\
\hline $5 \mathrm{~cm}$ & 0,7568 & B \\
\hline testigo & 0,4290 & $\mathrm{c}$ \\
\hline
\end{tabular}

En este caso se obtuvo el mismo resultado que en el análisis de covarianza; no obstante, aunque no se diferencian significativamente, el corte a $100 \mathrm{~cm}$ aparece con un valor más alto que el corte a menos de $5 \mathrm{~cm}$. Esto estaría indicando que en árboles más vigorosos, tales como lo son los de la clase de altura 2, las reservas de las fracciones de tallos gruesos, intermedios y finos serían aquellas que satisfacerían las demandas de respiración, de mantención y de cierto crecimiento invernal, produciéndose una diferencia con el corte a menos de $5 \mathrm{~cm}$, en que las únicas reservas de la planta provienen de la raíz (GARCIA, 1992). Con el corte a $50 \mathrm{~cm}$ de altura se obtuvo la mejor respuesta de la planta y el testigo presentó el menor crecimiento del período al igual que en todas las situaciones anteriores.

\section{Efecto del Tamaño Original de la Planta en la Biomasa del Rebrote}

El Cuadro 8 indica que sólo en la condición original, la productividad del rebrote obtenido en la clase de altura 2 se diferencia significativamente de la clase de altura 1 $(\mathrm{P}<=0,05)$. En la condición de rebrote no se produce esta diferenciación, aún cuando se detecta una tendencia similar al analizar los valores.

\section{Cuadro 8}

VARIACIÓN DEL PESO SECO DE LA BIOMASA DE Acacia saligna SEGÚN EL TAMAÑo ORIGINAL DE LA PLANTA PARA LA CONDICIÓN ORIGINAL Y SU REBROTE

\begin{tabular}{|c|c|cc|ccc|}
\hline $\begin{array}{c}\text { Clase de Altura } \\
(\mathbf{m})\end{array}$ & \multicolumn{3}{|c|}{$\begin{array}{c}\text { Condición Original } \\
\text { (kg/planta) }\end{array}$} & \multicolumn{3}{c|}{$\begin{array}{c}\text { Rebrote } \\
\text { (kg/planta) }\end{array}$} \\
\hline $1,25-1,80$ & 0,946 & $\mathrm{~A}$ & $\mathrm{a}$ & 0,813 & $\mathrm{~A}$ & $\mathrm{a}$ \\
\hline $1,81-3,50$ & 3,699 & $\mathrm{~B}$ & $\mathrm{a}$ & 1,439 & $\mathrm{~A}$ & $\mathrm{~b}$ \\
\hline
\end{tabular}

- Letras mayúsculas distintas en una misma columna indican diferencias significativas entre las clases de altura $(\mathrm{P}<=0,05)$.

- Letras minúsculas distintas en una misma línea indican diferencias significativas entre la planta original y su rebrote $(\mathrm{P}<=0,05)$. 
La diferenciación que se produce en la condición original para cada clase de altura es el resultado de la heterogeneidad que presentaba la plantación antes de la intervención, razón por la cual se analizó la información por clase de altura en forma independiente.

\section{Contenido de Humedad}

Se analizó el contenido de humedad de los 30 árboles intervenidos en la condición de rebrote. Al procesar los datos en forma independiente para cada clase de altura, la tendencia se mantiene; a mayor velocidad de crecimiento, menor contenido de humedad (Cuadro 9).

\section{Cuadro 9}

CONTENIDO DE HUMEDAD DEL REBROTE (\%)

\begin{tabular}{|c|c|c|c|}
\hline $\begin{array}{c}\text { C.Altura } \\
(\mathbf{m})\end{array}$ & Corte $(-5$ cm) & Corte $(50 \mathbf{~ c m})$ & Corte $(\mathbf{1 0 0} \mathbf{~ c m})$ \\
\hline $1,25-1,80$ & 176,8 & 132,98 & 155,68 \\
\hline $1,81-3,50$ & 211,34 & 161,46 & 161,70 \\
\hline Media & 194,07 & 147,22 & 158,69 \\
\hline
\end{tabular}

Según el Cuadro 9, en las dos clases de altura el corte a $50 \mathrm{~cm}$ presenta el menor contenido de humedad, intervención con la que se obtuvo la mayor productividad. Por otro lado, el corte bajo presentó el mayor contenido de humedad. Esto se explica porque a una mayor velocidad de crecimiento, la lignificación de la planta aumenta y el porcentaje de tejidos tiernos disminuye. En cambio, con un lento crecimiento, se mantiene una alta proporción de ramas finas o delgadas en la planta, conservando ésta un alto contenido de humedad.

Por otra parte, se consideró la relación entre el contenido de humedad de la biomasa obtenida en la condición original y su rebrote como respuesta a la intervención (Cuadro $10)$.

\section{Cuadro 10}

\section{CONTENIDO DE HUMEDAD (\%) PARA LA CONDICIÓN ORIGINAL Y EL REBROTE EN CADA CLASE DE ALTURA.}

\begin{tabular}{|c|c|c|}
\hline C. Altura & Original & Rebrote \\
\hline $1,25-1,80$ & 54,51 & 155,5 \\
\hline $1,81-3,50$ & 49,47 & 128,7 \\
\hline Media & $\mathbf{5 1 , 5 9}$ & $\mathbf{1 4 2 , 1}$ \\
\hline
\end{tabular}

Se observa un gran aumento en el contenido de humedad del rebrote con respecto a la condición original, lo que le da una mayor suculencia al material y aumenta su palatabilidad. 
En la condición original y la condición de rebrote se mantiene la tendencia de un mayor contenido de humedad en la clase de altura menor, debido principalmente a su lenta velocidad de crecimiento con respecto a la clase de altura mayor, y por lo tanto su menor lignificación.

Cabe destacar la magnitud del aumento del contenido de humedad obtenido con la intervención realizada. GARCIA (1992), para rebrote de Atriplex nummularia obtuvo aumentos, con respecto a la condición original, del orden de 15 a $30 \%$. En el caso de Acacia saligna los aumentos de contenido de humedad fueron de alrededor de $100 \%$ para la clase de altura menor y $80 \%$ para la clase de altura mayor.

\section{CONCLUSIONES}

El corte a $50 \mathrm{~cm}$ de altura resultó ser el tratamiento con el cual se logró el mayor crecimiento y vigor de los individuos para el período de ocho meses, independientemente de las dos clases de altura predefinidas.

En la clase de altura mayor se obtuvieron mejores resultados que en la clase de altura menor, de modo que individuos más vigorosos en un comienzo responden también en forma más vigorosa a la intervención.

La productividad, en material forrajero disponible para el ganado, aumenta considerablemente al efectuar el corte a temprana edad. Este aumenta el crecimiento, el vigor y la suculencia de la planta y además permite una mayor disponibilidad de forraje para el ganado, al bajar la altura del follaje del árbol.

Los contenidos de humedad obtenidos en el rebrote de cada tratamiento confirman que, a una mayor velocidad de crecimiento, disminuye el contenido de humedad, ya que la lignificación de los tejidos es más rápida.

La baja mortalidad de individuos producto de las intervenciones demuestra la buena reacción que presenta Acacia saligna frente a este tipo de manejo silvicultural.

\section{REFERENCIAS}

FAO. 1992. Reunión de la Red Latinoamericana de Cooperación Técnica en Dendroenergía y Constitución de la Red Brasileña en el tema. 58 p.

García, P. 1992. Efecto del corte en la producción y calidad forrajera del rebrote de Atriplex nummularia (Lindl.). Universidad de Chile. Facultad de Ciencias Agrarias y Forestales. Santiago. Tesis Ing. Forestal. Pág. 1-12-32.

Lailhacar, S. 1986. Recursos forrajeros utilizados en producción ovina. I Zona de clima mediterráneo árido y semiárido (secano comprendido entre los valles de Elqui y Aconcagua). En: García, G. (ed): Producción ovina. Universidad de Chile. Facultad de Ciencias Agrarias y Forestales. Departamento de Producción Animal. Pp 25-57. 
Vita, A. y Grez, I. 1992. Introducción de especies forestales combustibles y forrajeras en la IV Región. Evaluación 1990. Santiago. Universidad de Chile. Facultad de Ciencias Agrarias y Forestales - Corporación Nacional Forestal. 61 p.

Wrann, J. 1985. Metodología para el análisis de la regeneración natural en formaciones arbóreas nativas de la zona semiárida de Chile. En: $2^{\circ}$ Encuentro Regional CIID. América Latina y El Caribe. Santiago, Chile. 